University of Wollongong

Research Online

Australian Institute for Innovative Materials -

Papers

Australian Institute for Innovative Materials

2012

The role of unbound oligomers in the nucleation and growth of electrodeposited polypyrrole and method for preparing high strength, high conductivity films

\author{
Wen Zheng \\ University of Wollongong,wenz@uow.edu.au \\ Joselito M. Razal \\ University of Wollongong, jrazal@uow.edu.au \\ Geoffrey M. Spinks \\ University of Wollongong, gspinks@uow.edu.au \\ Van-Tan Truong \\ University of Wollongong \\ Philip G. Whitten \\ University of Wollongong, whitten@uow.edu.au
}

See next page for additional authors

Follow this and additional works at: https://ro.uow.edu.au/aiimpapers

Part of the Engineering Commons, and the Physical Sciences and Mathematics Commons

Research Online is the open access institutional repository for the University of Wollongong. For further information contact the UOW Library: research-pubs@uow.edu.au 


\title{
The role of unbound oligomers in the nucleation and growth of electrodeposited polypyrrole and method for preparing high strength, high conductivity films
}

\begin{abstract}
Polypyrrole is a material with immensely useful properties suitable for a wide range of electrochemical applications, but its development has been hindered by cumbersome manufacturing processes. Here we show that a simple modification to the standard electrochemical polymerization method produces polypyrrole films of equivalently high conductivity and superior mechanical properties in one-tenth of the polymerization time. Preparing the film as a series of electrodeposited layers with thorough solvent washing between layering was found to produce excellent quality films even when layer deposition was accelerated by high current. The washing step between the sequentially polymerized layers altered the deposition mechanism, eliminating the typical dendritic growth and generating nonporous deposits. Solvent washing was shown to reduce the concentration of oligomeric species in the near-electrode region and hinder the three-dimensional growth mechanism that occurs by deposition of secondary particles from solution. As artificial muscles, the high density sequentially polymerized films produced the highest mechanical work output yet reported for polypyrrole actuators.
\end{abstract}

\section{Keywords}

growth, oligomers, polypyrrole, method, preparing, high, strength, conductivity, nucleation, films, role, electrodeposited, unbound

\section{Disciplines}

Engineering | Physical Sciences and Mathematics

\section{Publication Details}

Zheng, W, Razal, JM, Spinks, GM, Truong, V, Whitten, PG \& Wallace, GG (2012), The role of unbound oligomers in the nucleation and growth of electrodeposited polypyrrole and method for preparing high strength, high conductivity films. Langmuir: The ACS Journal of Surfaces and Colloids, 28(29), pp. 10891-10897.

\section{Authors}

Wen Zheng, Joselito M. Razal, Geoffrey M. Spinks, Van-Tan Truong, Philip G. Whitten, and Gordon G. Wallace 


\section{The Role of Unbound Oligomers in the Nucleation and Growth of Electrodeposited Polypyrrole and Method for Preparing High Strength, High Conductivity Films}

Wen Zheng, ${ }^{\dagger}$ Joselito M. Razal, ${ }^{\dagger}$ Geoffrey M. Spinks, ${ }^{* \dagger}$ Van-Tan Truong, ${ }^{\ddagger}$ Philip G. Whitten, ${ }^{\dagger}$ and Gordon G. Wallace ${ }^{\dagger}$

${ }^{\dagger}$ Intelligent Polymer Research Institute, ARC Centre of Excellence for Electromaterials Science, AIIM Facility, Innovation Campus, University of Wollongong, Wollongong, NSW 2522 Australia

${ }^{\ddagger}$ Platforms Science Laboratory, Maritime Platforms Division, Defence Science and Technology Organization, Melbourne, VIC 3011, Australia

\section{Supporting Information}

ABSTRACT: Polypyrrole is a material with immensely useful properties suitable for a wide range of electrochemical applications, but its development has been hindered by cumbersome manufacturing processes. Here we show that a simple modification to the standard electrochemical polymerization method produces polypyrrole films of equivalently high conductivity and superior mechanical properties in onetenth of the polymerization time. Preparing the film as a series of electrodeposited layers with thorough solvent washing between layering was found to produce excellent quality films even when layer deposition was accelerated by high current. The washing step between the sequentially polymerized layers altered the deposition mechanism, eliminating the typical dendritic growth and generating nonporous deposits. Solvent washing was shown to reduce the concentration of oligomeric species in the near-electrode region and hinder the three-dimensional growth mechanism that occurs by deposition of secondary particles from solution. As artificial muscles, the high density sequentially polymerized films produced the highest mechanical work output yet reported for polypyrrole actuators.

\section{INTRODUCTION}

Artificial muscles, ${ }^{1-3}$ battery electrodes, ${ }^{4}$ and sensors ${ }^{5}$ that use conducting polymers such as polypyrrole (PPy) demand highly conductive and mechanically robust films or fibers for enhanced device performance. Most contemporary practitioners use an electrochemical method to prepare free-standing PPy films using a slow, low-temperature, galvanostatic polymerization of pyrrole monomer in organic solvent. ${ }^{6}$ Such methods produce highly conductive PPy films typically exceeding $200 \mathrm{~S} / \mathrm{cm}$ (depending upon dopant ion), although breaking strength can still be as low as a few MPa when operated electrochemically in preferred electrolytes. ${ }^{7}$ The slow polymerization is a major encumbrance with $8 \mathrm{~h}$ or more of polymerization time needed to generate practical film thickness. ${ }^{8,9}$ Although the rate of PPy synthesis can be easily accelerated by using higher deposition currents, previous attempts to utilize such high current densities $\left(2-4 \mathrm{~mA} / \mathrm{cm}^{2}\right.$, based on the geometrical area of the electrode) produced PPy films with inferior mechanical properties (elastic modulus $\sim 200 \mathrm{MPa})^{10}$ and low conductivity $(\sim 6 \mathrm{~S} / \mathrm{cm}){ }^{11}$ What is needed is a method to rapidly prepare strong and highly conductive PPy free-standing films.

A wide range of electrochemical procedures have been used to prepare PPy coatings and films with the polymer structure and properties strongly dependent upon the electrolyte composition, ${ }^{12}$ polymerization temperature, ${ }^{13}$ and electrodeposition conditions. ${ }^{14}$ Faster electrodeposition as occurs galvanostatically at higher current densities or potentiostatically at higher electrode potentials tends to produce rougher surfaces $^{15}$ and inferior properties. ${ }^{16}$ The surface roughness is largely determined by the polymer nucleation and deposition growth mechanisms. As in metal electrodeposition, ${ }^{17,18}$ nucleation can be either instantaneous or progressive and growth can be 2-dimensional or 3-dimensional. Both types of nucleation mechanisms have been identified in PPy electrodeposition and depend on the type of electrode used. ${ }^{19}$ Otero and De Larreta showed that smoother films were produced by potentiodynamic polymerization where the alternating anodic and cathodic cycles generated a greater nucleation density and hindered the 3-D growth of particles. In other studies, both 2-D and 3-D growth mechanisms have been identified for electropolymerized conducting polymers. ${ }^{20}$ The former mechanism favors a layer-by-layer deposition process and produces more compact deposits, while the continuous growth of nuclei in three dimensions produces porous films. Most studies of

Received: April 25, 2012

Revised: June 28, 2012

Published: July 13, 2012 


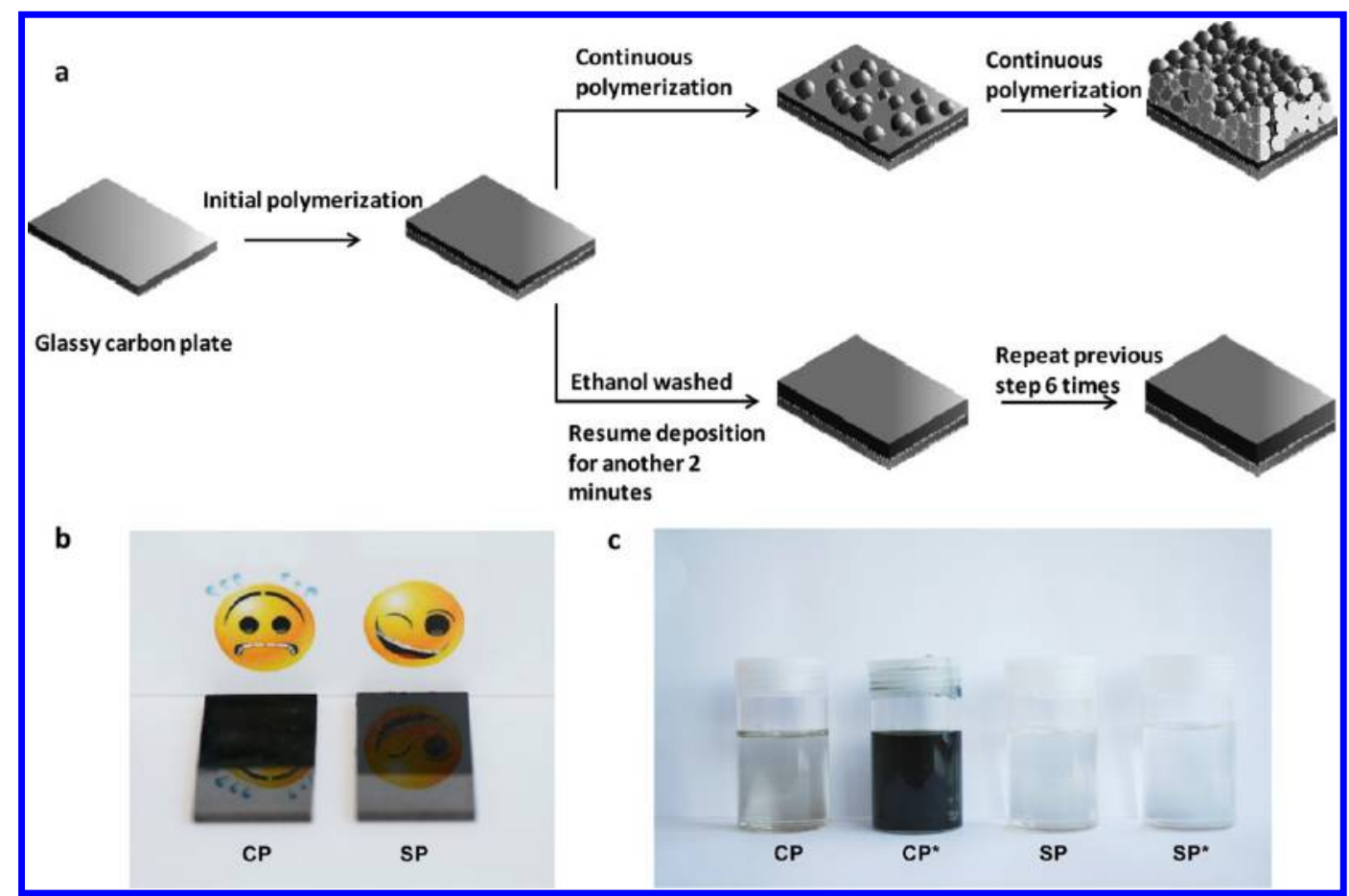

Figure 1. Preparation of SP and CP PPy films. (a) Schematic illustration of the polymerization process of PPy; top route: continuous polymerization $(\mathrm{CP})$ using $3 \mathrm{~mA} / \mathrm{cm}^{2}$ for $16 \mathrm{~min}$; bottom route: sequential polymerization (SP), using $3 \mathrm{~mA} / \mathrm{cm}^{2}$ where each layer of the 8 total layers was deposited for $2 \mathrm{~min}$. Ethanol wash and blow drying with nitrogen were applied between each SP PPy layer. (b) Photographs of PPy-coated, polished glassy carbon plates show a distinct interface between the PPy-coated (top) and uncoated (bottom) regions. The CP PPy film was rough with a nonreflective matte appearance while the SP PPy film was smooth, shiny and highly reflective. (c) Photographs of polymerization solutions that remained after polymer deposition by either SP or CP process. Aliquots from CP and SP processes were taken immediately after polymerization was completed. $\mathrm{CP}^{*}$ and $\mathrm{SP}^{*}$ are aliquots photographed seven days after the completion of polymerization.

electrodeposited conducting polymers indicate that the first formed layer is by 2-D nucleation and growth ${ }^{21}$ with $3-\mathrm{D}$ growth favored at longer polymerization times. ${ }^{22-24}$

The prior work described demonstrates the trade-off between PPy film properties and preparation time with slow polymerization required to produce high-quality films through a preference for progressive nucleation and 2-D growth. The modified synthesis method we report here significantly reduces the polymerization time required to fabricate free-standing PPy films from $8 \mathrm{~h}$ to just $16 \mathrm{~min}$ without compromising film properties. The method involves a process of sequentially depositing micrometer thick PPy layers with solvent washing between layers (Figure 1a) and is hereby referred to as sequential polymerization (SP). Herein we show that the SP films are highly conductive $(220 \mathrm{~S} / \mathrm{cm})$ with excellent mechanical properties (1.9 GPa modulus and $164 \mathrm{MPa}$ tensile strength) and can be used as high-performance artificial muscles.

\section{EXPERIMENTAL SECTION}

Reagents and Materials. Tetrabutylammonium hexafluorophosphate $\left(\mathrm{TBA} \cdot \mathrm{PF}_{6}\right.$, Fluka), propylene carbonate (PC, Sigma-Aldrich), and ethanol of HPLC grade (Ajax) were used as-received. Pyrrole (Fluka) was distilled before use. The polymerization solution contained $0.06 \mathrm{M}$ of pyrrole and $0.05 \mathrm{M}$ of $\mathrm{TBA}^{\mathrm{P}} \cdot \mathrm{PF}_{6}$ mixed in PC containing $1 \mathrm{wt} \%$ of deionized water. Before the reaction commenced, the whole solution was purged with nitrogen gas for $20 \mathrm{~min}$ and cooled to $-31{ }^{\circ} \mathrm{C}$. A $40 \mathrm{~mm} \times 20 \mathrm{~mm} \times 2 \mathrm{~mm}$ (length $\times$ width $\times$ thickness) glassy carbon working electrode (SIGRADUR Hochtemperatur-Werkstoffe $\mathrm{GmbH}$ ) was used as the substrate for electropolymerization of pyrrole. The counter electrode was reticulated vitreous carbon (RVC) foam ( $80 \mathrm{ppi})$.
Electrochemical Polymerization. PPy films were prepared using three different electrochemical processes as illustrated in Figure 1. SP films were deposited in eight steps with each step involving galvanostatic polymer electrodeposition at $3 \mathrm{~mA} / \mathrm{cm}^{2}$ for $2 \mathrm{~min}$, removal of the coated working electrode from the solution, washed in a stream of ethanol, dried with nitrogen gas, and returned to the solution. SP films (without washing) were prepared in an identical manner except that the ethanol wash was omitted. Continuously polymerized (CP) films were prepared in a one-step process involving polymer deposition at $0.1 \mathrm{~mA} / \mathrm{cm}^{2}$ for $8 \mathrm{~h}$ or $3 \mathrm{~mA} / \mathrm{cm}^{2}$ for $16 \mathrm{~min}$. Finally, for comparison purposes SP films (with washing) were also prepared at $+21{ }^{\circ} \mathrm{C}$. All other films were polymerized at $-31{ }^{\circ} \mathrm{C}$. A film thickness of $\sim 10 \mu \mathrm{m}$ was produced in each case, and the films could be readily peeled off the electrode surface.

Characterization. Electron microscopy was performed with a JEOL JSM-7500 FA. For examination of free-standing film cross sections, samples were mounted in epoxy and sectioned by ultramicrotome. The cross sections were imaged by scanning transmission electron microscopy (STEM) which provided maximum contrast between PPy and PPy-epoxy interface. Film thicknesses were measured from images of STEM cross sections. Mechanical properties of the PPy free-standing films in the dry state were measured with EZ$S$ universal testing machine (Shimadzu). All samples were cut into about $2 \mathrm{~mm}$ wide and $15 \mathrm{~mm}$ long, drawn at speed of $1 \mathrm{~mm} / \mathrm{min}$. The conductivity was measured by using a four-point probe (RM3, Jandel). Prior to those measurements, all PPy samples were carefully washed in ethanol to remove $\mathrm{PC}$ and then dried in a vacuum at $35^{\circ} \mathrm{C}$ overnight. The reported mechanical and electrical properties were obtained from four samples. Film densities were determined using a pycnometer.

Actuation tests for $\mathrm{PPy} \cdot \mathrm{PF}_{6}$ were performed in $0.25 \mathrm{M} \mathrm{TBA} \cdot \mathrm{PF}_{6} \mathrm{PC}$ electrolyte using a conventional three-electrode setup. The threeelectrode arrangement allows for the accurate control of the applied electrochemical potential with respect to a reference electrode. Accurate control of the electrochemical potential during actuation 
Table 1. Electronic Conductivity and Mechanical Properties of PPy Films Prepared by Either the Sequential Polymerization (SP) or Continuous Polymerization (CP) Method $^{a}$

\begin{tabular}{|c|c|c|c|c|c|}
\hline & $\mathrm{CP}: 0.1 \mathrm{~mA} / \mathrm{cm}^{2},-31{ }^{\circ} \mathrm{C}$ & $\mathrm{CP}: 3 \mathrm{~mA} / \mathrm{cm}^{2},-31{ }^{\circ} \mathrm{C}$ & $\mathrm{SP}:^{b} 3 \mathrm{~mA} / \mathrm{cm}^{2},-31{ }^{\circ} \mathrm{C}$ & $\mathrm{SP}:^{c} 3 \mathrm{~mA} / \mathrm{cm}^{2},-31{ }^{\circ} \mathrm{C}$ & $\mathrm{SP}:^{b} 3 \mathrm{~mA} / \mathrm{cm}^{2},+21^{\circ} \mathrm{C}$ \\
\hline elastic modulus (GPa) & $1.0 \pm 0.1$ & $0.34 \pm 0.03$ & $1.9 \pm 0.1$ & $0.26 \pm 0.01$ & $1.3 \pm 0.1$ \\
\hline tensile strength $(\mathrm{MPa})$ & $50 \pm 3$ & $4.3 \pm 1.9$ & $164 \pm 6$ & $2.4 \pm 0.7$ & $67 \pm 8$ \\
\hline elongation at break (\%) & $28 \pm 4$ & $4 \pm 0.6$ & $26 \pm 2$ & $3 \pm 1$ & $35 \pm 3$ \\
\hline $\begin{array}{l}\text { conductivity }(\mathrm{S} / \mathrm{cm}) \\
\text { density }\left(\mathrm{g} / \mathrm{cm}^{3}\right)\end{array}$ & $177 \pm 4$ & $\begin{array}{l}64 \pm 7 \\
1.27\end{array}$ & $\begin{array}{l}220 \pm 3 \\
1.64\end{array}$ & $50 \pm 3$ & $175 \pm 5$ \\
\hline
\end{tabular}

${ }^{a}$ Films were prepared galvanostatically at the shown current densities and temperature. Where reported, the error is a standard deviation. ${ }^{b}$ With ethanol solvent washing between sequentially deposited layers. ${ }^{c}$ Without ethanol solvent washing.

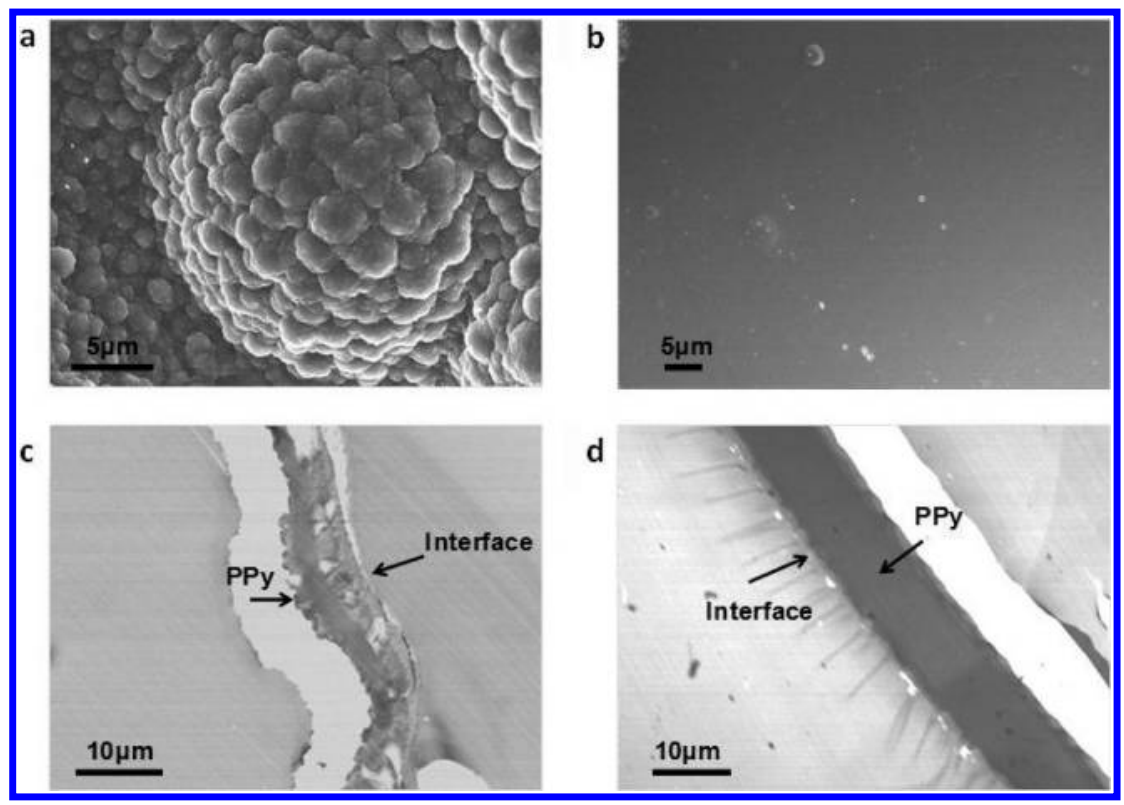

Figure 2. Morphology of SP and CP PPy films. (a, b) Scanning electron micrographs (SEM) showing the electrolyte surface and (c, d) scanning transmission electron micrographs (STEM) cross section of the PPy films. (a) and (c) are for CP and (b) and (d) are for SP films. All samples were polymerized at a current density of $3 \mathrm{~mA} / \mathrm{cm}^{2}$ and at $-31{ }^{\circ} \mathrm{C}$.

testing is important to control the redox reactions responsible for PPy actuation and to prevent degradation by overoxidation. During the actuation tests, when potential was applied, the change in length at a fixed applied force was measured by a lever arm dual mode system (305B, Aurora Scientific Pty). When actuation tests were conducted under loads, the actuation strain was measured at the lowest load, and then the load was increased stepwise and the actuation test repeated at each load increment. The process was repeated until failure of the sample occurred.

\section{RESULTS AND DISCUSSION}

Significant improvements in both electrical and mechanical properties were evident from PPy films prepared by the SP method in comparison to films made by the CP method (Table 1). When prepared at the same high current density the SP films showed an astonishing 40 times increase in the breaking strength (160 MPa, Figure S1a) compared to the CP films (4 $\mathrm{MPa}$ ). Similarly, the elastic modulus and conductivity of the SP films (1.9 GPa and $220 \mathrm{~S} / \mathrm{cm}$, respectively) were significantly higher than the CP films (0.34 GPa and $60 \mathrm{~S} / \mathrm{cm}$ ). Even in comparison to the standard low current density CP method (slow polymerization), the films prepared using the rapid SP method displayed superior tensile strength (3 times higher), modulus (nearly 2 times higher), and conductivity (30\% higher). Notably, these significantly improved properties were achieved in less than $5 \%$ of the polymerization time.
Further investigation of the properties obtained from SP films showed that the washing step and polymerization temperature were critically important. SP films prepared without washing had nearly identical mechanical and electrical properties to the CP films using the same high current density (Table 1). Polymerization temperature also had a significant effect with SP films (with washing) prepared at room temperature showing inferior properties to the equivalent films prepared at $-31^{\circ} \mathrm{C}$. Interestingly, the room temperature SP films were still superior to the CP films prepared at $-31^{\circ} \mathrm{C}$ and at the same current density.

The first clue to the origin of the improved properties of the rapidly produced SP films was their strikingly different visual appearance and microstructure. Both sides of the SP films prepared with solvent washing and at $-31{ }^{\circ} \mathrm{C}$ were shiny, smooth, and lustrous (Figures $1 \mathrm{~b}$ and $2 \mathrm{~b}$ ). A similar shiny appearance was noted for the CP films prepared slowly at a low current density. In contrast, the high current density CP films showed a rough and dull surface and the classical "cauliflower" morphology ${ }^{25-27}$ on the electrolyte side (Figures $1 \mathrm{~b}$ and $2 \mathrm{a}$ ). Cross-section scanning transmission electron microscope (STEM) images show a highly porous structure with variable thickness $(6-10 \mu \mathrm{m})$ for the high-current CP film (Figure 2c) and a homogeneously dense, uniformly thick $(\sim 10 \mu \mathrm{m})$ structure for the SP film (Figure 2d). Density measurements confirmed the porous nature of the CP films $\left(1.27 \mathrm{~g} / \mathrm{cm}^{3}\right)$ 


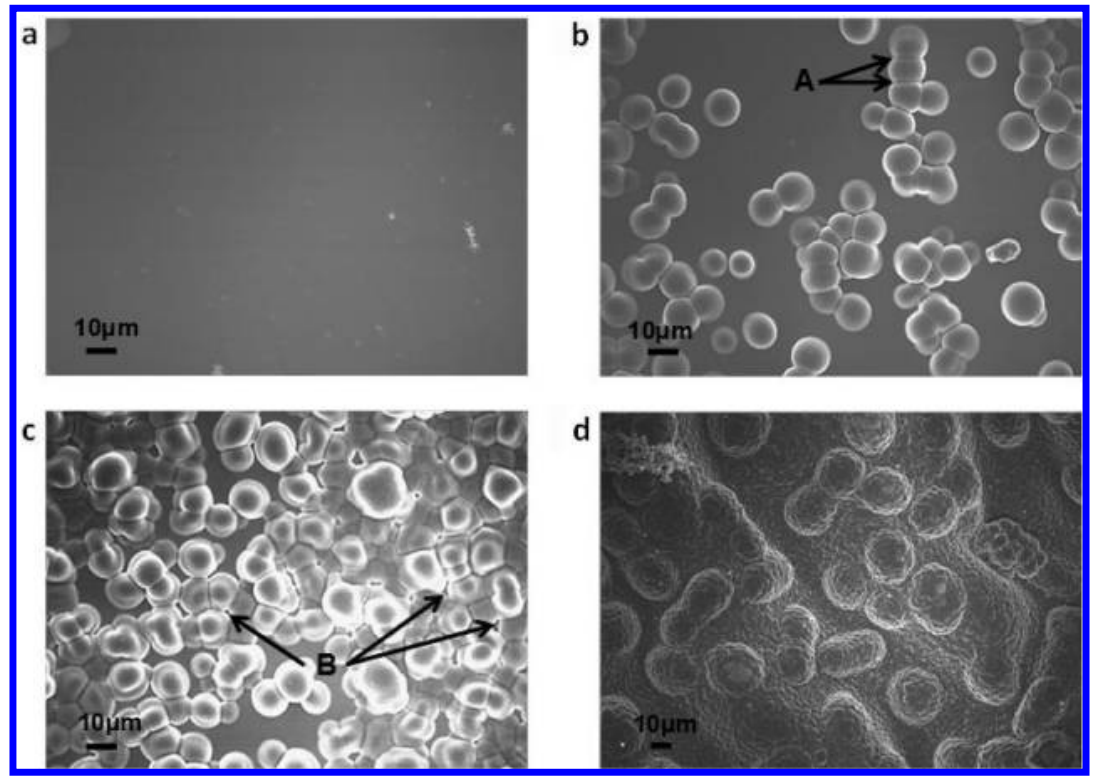

Figure 3. Evolution of surface morphology of CP PPy films at high current density. Surface features of the electrolyte side of CP PPy film after continuous polymerization for different times: (a) 2, (b) 4, (c) 8, and (d) $16 \mathrm{~min}$.

compared with the SP films $\left(1.64 \mathrm{~g} / \mathrm{cm}^{3}\right)$. To the authors' knowledge, the highest previously reported density for PPy was $1.44 \mathrm{~g} / \mathrm{cm}^{3} .^{28}$ The high degree of porosity, microstructural, and molecular defects, including molecular species not connected to the cross-linked polymer network, and rough surface account for the low mechanical strength and modulus of the CP films, as the pores and internal defects act as stress concentrators or plasticizers. ${ }^{16,29,30}$ The higher conductivity of the SP films likely arise from increased conjugation length, as no difference in long-range order or chemical composition between SP and CP films could be detected by X-ray diffraction (Figure S1b) or Raman spectroscopy (Figure S1d). Studies of electropolymerized polythiophene films noted decreases in conductivity and density with increasing film thickness and the enhanced conductivity of thin films was attributed to a longer chain conjugation length. ${ }^{31,32}$ It is also noted that the tortuous conduction pathway in porous carbon means that conductivity scales directly with density. ${ }^{33}$ It follows that the less dense, porous CP films will show a reduced conductivity than the denser SP films.

SEM imaging at different stages of high current CP reveal important details of the deposition mechanisms and the source of microscale surface roughening and internal porosity. Examination of the CP film after 2 min polymerization showed a homogenously smooth surface (Figure 3a). However, a separately prepared CP film polymerized for 4 min revealed numerous smooth, almost spherical particles $(10-15 \mu \mathrm{m}$ in diameter), which probably deposited on top of the first-formed PPy layer (i.e., as formed in the first $2 \mathrm{~min}$ ) (Figure 3b). In some instances, a flat boundary was evident between impinging particles (labeled A in Figure 3b), indicating that the particles first nucleate and then grow radially with time. The surface concentration of these "secondary particles" increased with polymerization duration such that the underlying, first-formed PPy layer was almost completely covered with particles after continuous polymerization for $8 \mathrm{~min}$ (Figure $3 \mathrm{c}$ ). Of note was the occurrence of porosity that resulted from incompletely fused particles in regions of high particle concentration (labeled $\mathrm{B}$ in Figure 3c). Eventually, the well-known "cauliflower" pattern was evident in the 16 min CP film (Figure 3d) with smaller particles forming on the surfaces of the original particles. Since the washed SP film (prepared at the same temperature and equivalent total polymerization time of 16 min) developed no such porosity or "cauliflower" surface roughness (Figure 2b), it is evident that the first-formed smooth PPy layer was deposited in each sequential step without the formation of secondary particles. The sequentially polymerized film prepared without the washing step had a similarly rough surface structure (Figure S2a) as the rapidly polymerized CP film. Clearly, the washing step prevented the nucleation and growth of secondary particles.

These observations of the PPy film morphology development are consistent with previous studies of the nucleation and growth of electropolymerized conducting polymers. The buildup of oligomeric species near the electrode has been implicated in the 3-D growth mechanism of electrodeposited conducting polymers. At the point of supersaturation of oligomers in solution, clusters deposit and create growing nuclei. ${ }^{34}$ The growth of the 3-D nuclei is favored because of their relatively higher surface area. The deposition kinetics are determined by the reaction conditions, charge transfer and mass transfer rates, and oligomer solubility. Within the resolution limits of the SEM images of Figure 3, it is evident that initial deposition of the PPy occurs by 2-D nucleation and growth followed by progressive nucleation and 3-D growth of secondary particles resulting from precipitation of oligomer clusters.

Analysis of the ethanol wash solutions and polymerization solutions confirms that high concentrations of pyrrole oligomeric species are related to the formation of the secondary particles. The $480 \mathrm{~nm}$ absorption peak observed in the ultraviolet-visible spectra of all ethanol wash solutions indicates the presence of pyrrole oligomers. ${ }^{35}$ Similarly, oligomers were found in the polymerization solution following the continuous deposition but were not detected in the polymerization solution after SP (Figure S3). Furthermore, the $\mathrm{CP}$ polymerization solution gradually darkened upon standing for 1 week with no applied current or potential, whereas the SP 
polymerization solution remained colorless (Figure 1c). During electropolymerization, oligomers are produced and released into the electrolyte, ${ }^{36}$ and a high concentration of these more active species ${ }^{37,38}$ in the near-electrode solution promotes the heterogeneous nucleation and growth of secondary polymer particles. $^{39}$ Radical coupling of two oligomers significantly increases molecular weight, chain branching, and cross-linking, thereby reducing solubility ${ }^{40}$ and promoting precipitation. These conditions arise after $2 \mathrm{~min}$ of continuous polymerization at $3 \mathrm{~mA} / \mathrm{cm}^{2}$ and $-31{ }^{\circ} \mathrm{C}$. When reaction is slowed by a low current ${ }^{6}$ or a pulsed potential ${ }^{41}$ or is interrupted with a washing step, the oligomer concentration remains sufficiently low to prevent the formation of secondary particles. Without such particle formation, the separate layers deposited during SP were almost indistinguishable by cross-sectional STEM (Figure $2 \mathrm{~d}$ and Figure S2b). It is also possible that some oligomers remaining in the washed PPy deposits participate in the subsequent polymerization to encourage strong adhesion and assimilation of the layers.

The new SP PPy films were evaluated as artificial muscles since high conductivity and high strength are advantageous in these applications. Reversible oxidation and reduction of the PPy (Figure 4a) induces swelling and contraction that can perform mechanical work. The SP film had a sharper oxidation peak centered at a lower oxidation potential than the CP film, which is similar to that observed for PPy formed under very low

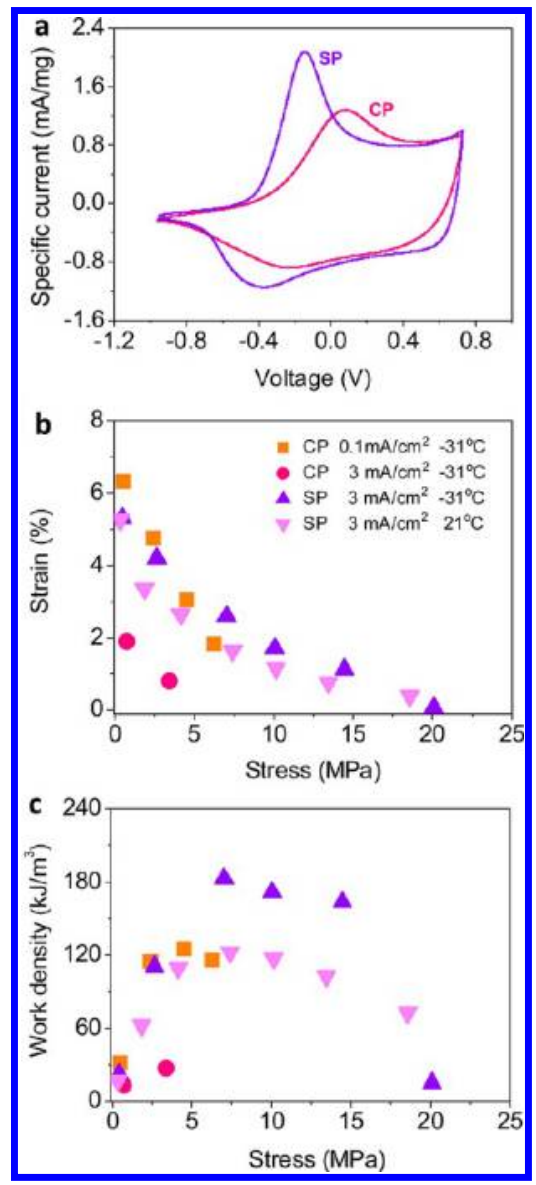

Figure 4. Artificial muscle performance: (a) cyclic voltammograms for $\mathrm{CP}$ and washed SP PPy films prepared at $3 \mathrm{~mA} / \mathrm{cm}^{2}$ and $-31{ }^{\circ} \mathrm{C}$; (b) actuation strain; and (c) work-per-cycle for isotonic actuation testing at different applied stress for various PPy films. current densities. ${ }^{42}$ All samples produced a positive actuation strain (percent change in length) when the applied electrochemical potential was ramped between -1.0 and $+0.8 \mathrm{~V}$ (versus a silver/silver ion reference electrode). The actuation strain diminished in magnitude with an increase in the isotonic tensile stress applied to the films (Figure 4b), in accord with previous studies of conducting polymer actuators. ${ }^{9}$ The free stroke (strain at zero applied stress) estimated by extrapolation was $\sim 6 \%$ for both washed SP films (prepared at -31 and 22 ${ }^{\circ} \mathrm{C}$ ) and the CP film prepared at low current density. The CP film prepared at higher current density had a considerably lower free stroke of $\sim 2 \%$ and reduced electroactivity, as evident from the broad and less pronounced oxidation and reduction peaks obtained from cyclic voltammetry (Figure 4a). The decreased charge transfer efficiency of this CP film is likely related to increased voltage drop (IR drop) along PPy sample $\mathrm{e}^{43}$ as a result of the lower conductivity. The CP film prepared at a high current density was also extremely fragile (stress at break $<4$ $\mathrm{MPa}$ ) when it was saturated with the PC electrolyte. In contrast, the washed SP film prepared at $-31{ }^{\circ} \mathrm{C}$ sustained stresses up to $20 \mathrm{MPa}$ without failure during actuation work cycles. The high load capability of this rapidly polymerized SP films led to a high work-per-cycle of $190 \mathrm{~kJ} / \mathrm{m}^{3}$ at an applied stress of $8 \mathrm{MPa}$ (Figure 4c). This work density is higher than the previously highest reported value of $140 \mathrm{~kJ} / \mathrm{m}^{3}$ from PPy actuators that took $\sim 15$ times longer to be prepared. ${ }^{44}$

\section{CONCLUSIONS}

In summary, a simple route to rapidly prepare high quality PPy films and their superior performance as artificial muscles is reported. It is shown that solvent washing at regular intervals during the electrodeposition of PPy removes unbound oligomeric species from the deposited PPy and hinders secondary precipitation of polymer particles. Pore-free PPy deposits are then produced. The improved mechanical and electrical properties of these films can be directly related to their reduced porosity and reduction in microstructural and molecular defects. It is anticipated that the sequential polymerization-washing procedure could be readily automated to facilitate commercial-scale production. The higher strength, modulus, conductivity, and electroactivity of the sequentially produced PPy will cater for many applications requiring freestanding films. In future work, film synthesis conditions will be optimized by considering the effects of deposition current density, polymerization time for each layer, and polymerization temperature.

\section{ASSOCIATED CONTENT}

\section{Supporting Information}

Further information relating to the structural characterization of the prepared polypyrrole films. This material is available free of charge via the Internet at http://pubs.acs.org.

\section{AUTHOR INFORMATION}

\section{Corresponding Author}

*E-mail gspinks@uow.edu.au; Tel +61 24221 3010; Fax +61 2 42213112.

\section{Author Contributions}

The manuscript was written through equal contributions of all authors. All authors have given approval to the final version of the manuscript. 


\section{Notes}

The authors declare no competing financial interest.

\section{ACKNOWLEDGMENTS}

This work is funded by the Australian Research Council (ARC) and the Defence Science Technology Organisation (Australia). The authors thank Tony Romeo for obtaining the electron microscope images, Dr. Klaudia Wagner for valuable discussions, the Australian Research Council (ARC) and the Defence Science Technology Organisation (Australia) for financial support, and the ANFF Materials Node for their provision of research facilities. J.M.R., G.M.S., and G.G.W. were supported by ARC APD Fellowship, ARC Professorial Fellowship, and ARC Federation Fellowship, respectively.

\section{ABBREVIATIONS}

PPy, polypyrrole; $\mathrm{TBA} \cdot \mathrm{PF}_{6}$, tetrabutylammonium hexafluorophosphate; SP, sequential polymerization; $\mathrm{CP}$, continuously polymerized; STEM, scanning transmission electron microscopy; PC, propylene carbonate; RVC, reticulated vitreous carbon.

\section{REFERENCES}

(1) Baughman, R. H. Conducting Polymer Artificial Muscles. Synth. Met. 1996, 78, 339-353.

(2) Jager, E. W. H.; Smela, E.; Inganas, O. Microfabricating Conjugated Polymer Actuators. Science 2000, 290, 1540-1545.

(3) Smela, E. Conjugated Polymer Actuators for Biomedical Applications. Adv. Mater. 2003, 15, 481-494.

(4) Kakuda, S.; Momma, T.; Osaka, T.; Appetecchi, G. B.; Scrosati, B. Ambient-Temperature, Rechargeable, All Solid Lithium Polypyrrole Polymer Battery. I. Electrochem. Soc. 1995, 142, L1-L2.

(5) Shoa, T. N.; Madden, J. D. W.; Mirfakhrai, T.; Alici, G.; Spinks, G. M.; Wallace, G. G. Electromechanical Coupling in Polypyrrole Sensors and Actuators. Sens. Actuators, A 2010, 161, 127-133.

(6) Yoon, C. O.; Reghu, M.; Moses, D.; Heeger, A. J. Transport Near the Metal-insulator-transition-polypyrrole Doped with $\mathrm{PF}(6)$. Phys. Rev. B 1994, 49, 10851-10863.

(7) Ding, J.; Liu, L.; Spinks, G. M.; Zhou, D.; Wallace, G. G.; Gillespie, J. High Performance Conducting Polymer Actuators Utilising a Tubular Geometry and Helical Wire Interconnects. Sunth. Met. 2003, 138, 391-398.

(8) Madden, J. D.; Cush, R. A.; Kanigan, T. S.; Hunter, I. W. Fast Contracting Polypyrrole Actuators. Synth. Met. 2000, 113, 185-192.

(9) Spinks, G. M.; Liu, L.; Zhou, D.; Wallace, G. G. Strain Response from Polypyrrole Actuators Under Load. Adv. Funct. Mater. 2002, 12 (6-7), 437-440.

(10) Satoh, M.; Kaneto, K.; Yoshino, K. Dependences of Electrical and Mechanical Properties of Conducting Polypyrrole Films on Conditions of Electrochemical Polymerization in an Aqueous Medium. Sunth. Met. 1986, 14, 289-296.

(11) Dyreklev, P.; Granstrom, M.; Inganas, O.; Gunaratne, L.; Senadeera, G. K. R.; Skaarup, S.; West, K. The Influence of Polymerization Rate on Conductivity and Crystallinity of Electropolymerized Polypyrrole. Polvmer 1996, 37, 2609-2613.

(12) Otero, T. F.; Rodriguez, J. Role of Protons on The Electrochemical Polymerization of Pyrrole from Acetonitrile Solutions. I. Electroanal. Chem. 1994, 379, 513-516.

(13) Liang, W. B.; Lei, J. T.; Martin, C. R. Effect of Synthesis Temperature on the Structure, Doping Level and Charge Transport Properties of Polypyrrole. Synth. Met. 1992, 52, 227-239.

(14) Maddison, D. S.; Roberts, R. B.; Unsworth, J. ThermoelectricPower of Polypyrrole. Synth. Met. 1989, 33, 281-287.

(15) Patois, T.; Lakard, B.; Monney, S.; Roizard, X.; Fievet, P. Characterization of the Surface Properties of Polypyrrole Films:
Influence of Electrodeposition Parameters. Synth. Met. 2011, 161, $2498-2505$

(16) Gandhi, M.; Spinks, G. M.; Burford, R. P.; Wallace, G. G. Film Substructure and Mechanical Properties of Electrochemically Prepared Polypyrrole. Polvmer 1995, 36, 4761-4765.

(17) Naylor, A. J.; Koukharenko, E.; Nandhakumar, I. S.; White, N. M. Surfactant-Mediated Electrodeposition of Bismuth Telluride Films and its Effect on Microstructural Properties. Langmuir 2012, 28, $8296-8299$.

(18) Younes, O.; Zeiri, L.; Efrima, S.; Deutsch, M. Two- to Threedimensional Hecker Transitions in the Interfacial Electrodeposition of Silver. Langmuir 1997, 13, 1767-1772.

(19) Hwang, B. J.; Santhanam, R; Lin, Y. L. Nucleation and Growth Mechanism of Electroformation of Polypyrrole on a Heat-treated Gold/Highly Oriented Pyrolytic Graphite. Electrochim. Acta 2001, 46, 2843-2853.

(20) Li, F.-B.; Albery, W. J. Electrochemical Deposition of a Conducting Polymer, Poly(thiophene-3-acetic acid): The First Observation of Individual Events of Polymer Nucleation and TwoDimensional Layer-by-Layer Growth. Langmuir 1992, 8, 1645-1653.

(21) Wang, Y.; Northwood, D. O. An Investigation into the Nucleation and Growth of an Electropolymerized Polypyrrole Coating on a 316L Stainless Steel Surface. Thin Solid Films 2008, 516, 74277432.

(22) Schrebler, R.; Grez, P.; Cury, P.; Veas, C.; Merino, M.; Gomez, H.; Cordova, R.; del Valle, M. A. Nucleation and Growth Mechanisms of Poly(thiophene). Part 1. Effect of Electrolyte and Monomer Concentration in Dichloromethane. I. Electroanal. Chem. 1997, 430, 77-90.

(23) Hwang, B. J.; Santhanam, R.; Lin, Y. L. Nucleation and Growth Mechanism of Electropolymerization of Polypyrrole on Gold/Highly Oriented Pyrolytic Graphite Electrode. I. Electrochem. Soc. 2000, 147, 2252-2257.

(24) Tamburri, E.; Orlanducci, S.; Toschi, F.; Terranova, M. L.; Passeri, D. Growth Mechanisms, Morphology, and Electroactivity of PEDOT Layers Produced by Electrochemical Routes in Aqueous Medium. Synth. Met. 2009, 159, 406-414.

(25) Mitchell, G. R.; Geri, A. Molecular Organization of Electrochemically Prepared Conducting Polypyrrole Films. I. Phys. D: Appl. Phvs. 1987, 20, 1346-1353.

(26) Hara, S.; Zama, T.; Takashima, W.; Kaneto, K. Tris(trifluoromethylsulfonyl)methide-Doped Polypyrrole as a Conducting Polymer Actuator with Large Electrochemical Strain. Synth. Met. 2006, 156, 351-355.

(27) Kaynak, A. Effect of Synthesis Parameters on the Surface Morphology of Conducting Polypyrrole Films. Mater. Res. Bull. 1997, $32,271-285$.

(28) Lei, J. T.; Cai, Z. H.; Martin, C. R. Effect of Reagent Concentrations Used to Synthesize Polypyrrole on the Chemical Characteristics and Optical and Electronic-Properties of the Resulting Polymer. Synth. Met. 1992, 46, 53-69.

(29) Buckley, L. J.; Roylance, D. K.; Wnek, G. E. Influence of Sopant Ion and Synthesis Variables on Mechanical Properties of Polypyrrole Films. I. Polvm. Sci. Part B: Polvm. Phvs. 1987, 25, 2179-2188.

(30) Sutton, S. J.; Vaughan, A. S. On the Morphology and Growth of Electrochemically Polymerized Polypyrrole. Polvmer 1995, 36, 18491857.

(31) Roncali, J.; Yassar, A.; Garnier, F. Electrosynthesis of Highly Conducting Poly(3-methylthiophene) Thin Flims. I. Chem. Soc. Chem. Commun. 1988, 9, 581-582.

(32) Masuda, H.; Tanaka, S.; Kaeriyama, K. Electrochemical Polymerization of 3-dodecylthiophene-Effect of Current Density and Film Thickness. Synth. Met. 1989, 31, 29-36.

(33) Lu, X. P.; Nilsson, O.; Fricke, J.; Pekala, R. W. Thermal and Electrical Conductivity of Monolithic Carbon Aerogels. I. Appl. Phvs. 1993, 73, 581-584.

(34) Schrebler, R.; Grez, P.; Cury, P.; Veas, C.; Merino, M.; Gomez, H.; Cordova, R.; Del Valle, M. A. Nucleation and Growth Mechanisms of Poly(thiophene). Part 1. Effect of Electrolyte and Monomer 
Concentration in Dichloromethane. J. Electroanal. Chem. 1997, 430, 77-90.

(35) Zotti, G.; Martina, S.; Wegner, G.; Schluter, A. D. Well Defined Pyrrole Oligomers-Electrochemical and UV Vis Studies. Adv. Mater. 1992, 4, 798-801.

(36) Baker, C. K.; Reynolds, J. R. Use of the Quartz Microbalance in the Study of Polyheterocycle Electrosynthesis. Sunth. Met. 1989, 28, C21-C26.

(37) Wang, Y.; Tran, H. D.; Kaner, R. B. Applications of Oligomers for Nanostructured Conducting Polymers. Macromol. Rapid Commun. 2011, 32, 35-49.

(38) Tran, H. D.; Wang, Y.; D’Arcy, J. M.; Kaner, R. B. Toward an Understanding of the Formation of Conducting Polymer Nanofibers. ACS Nano 2008, 2, 1841-1848.

(39) Wang, Y.; Tran, H. D.; Liao, L.; Duan, X. F.; Kaner, R. B. Nanoscale Morphology, Dimensional Control, and Electrical Properties of Oligoanilines. I. Am. Chem. Soc. 2010, 132, 10365-10373.

(40) Sadki, S.; Schottland, P.; Brodie, N.; Sabouraud, G. The Mechanisms of Pyrrole Electropolymerization. Chem. Soc. Rev. 2000, 29, 283-293.

(41) Otero, T. F.; Delarreta, E. Electrochemical Control of the Morphology, Adherence, Appearance and Growth of Polypyrrole Films. Sunth. Met. 1988, 26, 79-88.

(42) Zhou, M.; Pagels, M.; Geschke, B.; Heinze, J. Electropolymerization of Pyrrole and Electrochemical Study of Polypyrrole. 5. Controlled Electrochemical Synthesis and Solid-state Transition of Well-defined Polypyrrole Variants. I. Phvs. Chem. B 2002, 106, 1006510073.

(43) Hutchison, A. S.; Lewis, T. W.; Moulton, S. E.; Spinks, G. M.; Wallace, G. G. Development of Polypyrrole-based Electromechanical Actuators. Proc. SPIE-Int. Soc. Opt. Eng. 1999, 3669, 242-253.

(44) Kaneto, K.; Fujisue, H.; Kunifusa, M.; Takashima, W. Conducting Polymer Soft Actuators Based on Polypyrrole Films Energy Conversion Efficiency. Smart Mater. Struct. 2007, 16, S250S255. 